\title{
Condensed SFEs for nonlinear mechanical problems
}

\author{
Antoine Llau ${ }^{\mathrm{a}, \mathrm{b}, \mathrm{c}}$, Julien Baroth ${ }^{\mathrm{c}, \mathrm{d}}$, Ludovic Jason ${ }^{\mathrm{a}, \mathrm{b}}$, Frédéric Dufour ${ }^{\mathrm{c}, \mathrm{d}}$ \\ ${ }^{a}$ SEMT, CEA DEN, Université Paris Saclay, 91191 Gif sur Yvette Cedex, France \\ ${ }^{b} I M S I A, C E A, C N R S$, EDF, ENSTA Paristech, Université Paris Saclay, 91762 Palaiseau Cedex, France \\ ${ }^{c}$ Univ. Grenoble-Alpes, 3SR, 38041 Grenoble Cedex 9, France \\ ${ }^{d}$ CNRS, 3SR, 38041 Grenoble Cedex 9, France
}

\begin{abstract}
This paper introduces a coupled approach between stochastic finite element methods and an adaptive condensation technique for the analysis of nonlinear mechanical problems under uncertainties. This coupling reduces the size of each individual nonlinear problem solved in SFE by the use of an adaptive condensation method. The reduced stiffnesses and other quantities necessary for the condensation technique are approximated using a second, low-order, polynomial expansion, thus taking advantage of the coupling with SFE. This approach also features a semi-analytical technique to compute accurately distributions of structural quantities of interest. This method is applied on an elasto-plastic steel bar with a small defect, and on a damaged beam under 4-point bending. In both cases it predicts the random behaviour of the structure quite accurately, and is able to provide higher-order models than a state-of-the-art stochastic collocation method, for a reduced computation time.
\end{abstract}

Keywords: Stochastic finite elements, Concrete cracking, Collocation, Static condensation, Metamodels.

\section{Introduction}

During the last decades, numerous methods have been developed, inside the general framework of stochastic finite elements (SFE). These methods allow the use of finite element analysis on uncertain mechanical problems. They can also quantify the influence on the structure of these uncertainties on quantities of interest. The Monte-Carlo (MC) method [1, 2] should be considered as the first one, and is still widely used in different application domains. However, more recent modeling techniques offer, compared to the MC method, higher orders of convergence, and require a reduced number of deterministic simulations $[3,4,5,6]$. These techniques are now used in several industrial simulation codes $[7,8]$. In certain cases, they even provide directly approximations of the statistical quantities of interest $[9,10,11]$ : mean values, statistical moments, probability density functions (PDFs), etc. SFE methods are characterized by three combined building blocks, which allow to predict the behaviour of a structure under uncertainties [6]

- A model for uncertainties (e.g. modeling of random material properties).

- A mathematical technique to solve deterministic problems (typically, direct FE simulation). 
- An algorithm to propagate deterministic solutions depending on the uncertainties (e.g. perturbation, polynomial chaos expansion).

Various methods have been developed to model the uncertainties on geometry, material properties, loading. Techniques to propagate uncertainties through deterministic solutions have also been widely studied, and various classes of methods are now available in literature. However, in the framework of SFE, few studies addressed the problem of the model and of the numerical techniques to solve the deterministic problems, in particular to minimize the computational cost $[6,12]$. It is especially the case for large scale nonlinear systems, for which non intrusive approaches such as Polynomial-Chaos or collocation-based methods are generally preferred to intrusive ones.

This work presents a coupled method to solve nonlinear SFE problems at a reduced cost. It includes a deterministic system reduction approach similar to those in [13] (using dynamic condensation) or in [14] (using static condensation). However, the presented method :

- uses an adaptive condensation technique based on a two-level Guyan's reduction, suitable for nonlinear mechanical problems $[15,16]$.

- builds a second, lower-order, metamodel. This is used for the stiffnesses, equivalent loadings and displacements fields necessary in the condensation technique.

- provides full probability density functions (PDF) of quantities of interest thanks to a semi-analytical approach or Monte-Carlo simulations when it is not possible.

This method may be used in mechanical engineering, to evaluate the robustness of numerical models, build fragility curves of mechanical systems, etc. In particular, an application to civil engineering structures is proposed in this contribution.

Firstly, the nonlinear stochastic mechanical problem to be solved is presented and formulated. Then, the proposed method to solve it efficiently is described. A validation is performed on an heuristic test case, where the presented SFEM is compared to analytical results. Finally, an application is presented, where the method is compared to a state-of-the-art SFE technique.

\section{Problem setting}

\subsection{Stochastic nonlinear mechanical problem}

The problem is a general $n$-dimensional nonlinear mechanical problem, defined on a bounded domain $\Omega \in \mathbb{R}^{d}(d=1,2,3)$. It is characterized by a set of $r$ input parameters $y=\left(y_{1}, \ldots, y_{r}\right)$. It is assumed that the problem is well posed. The problem is solved using the finite element method (implemented in Cast3M [17]). 
The resulting $n$-dimensional mechanical system is considered through a pseudo-time $t$. Using a discretized time $\tau$, the problem can be solved iteratively :

$$
\left[K^{\tau}\left(y, u^{\tau}(y)\right)\right] \cdot\left[u^{\tau}(y)\right]=\left[F^{\tau}(y)\right]
$$

where $K^{\tau} \in \mathbb{R}^{n, n}$ is the stiffness matrix, $u^{\tau} \in \mathbb{R}^{n}$ the nodal displacement vector, and $F^{\tau} \in \mathbb{R}^{n}$ the nodal force vector (at time $t=\tau$ ). Mechanical quantities of interest can be extracted once the structural problem has been solved: they are considered as a random vector $Z=h^{\tau}(y)$. We consider in the following that the random input parameters can be normalized to a standardized Gaussian random vector $x=\left(x_{1}, \ldots, x_{r}\right)=T(y)$, using the Gaussian standardization function $T$ [18]. Given that any distribution can be generated from a standard distribution [18], this methodology allows to chose any type of law for the actual r.v.s used in the problem (mechanical properties, random fields, etc) with a single SFE method. The denormalization function is therefore included in the mechanical response function $\mathcal{M}$. The random vector $Z$ writes :

$$
Z=h^{\tau}(y)=h^{\tau} \circ T^{-1}(x)=\mathcal{M}(x)
$$

\subsection{Construction of a metamodel}

The input uncertain parameters $y$ are represented using a $r$-dimensional vector of standard independent random variables (r.v.s) $X=T(Y)=\left(X_{1}, \ldots, X_{r}\right)$, defined on the probability space $(\Theta, \mathcal{F}, \mathcal{P})$.

In some cases, the function $\mathcal{M}$ is approximated using an analytical function $\tilde{Z}=\tilde{\mathcal{M}}(X): \Omega \rightarrow I$. This approximation $\tilde{\mathcal{M}}$ is classically provided using SFE methods under the general form of a metamodel (or surface response). This metamodel is often a projection of the mechanical function $\mathcal{M}$ onto a suitable basis of an $n_{g}$ dimensional domain of orthogonal polynomials $\Phi_{p}, p=1, \ldots, n_{g}$. The approximation of the random variable (r.v.) writes :

$$
Z=\mathcal{M}(X) \simeq \tilde{Z}=\tilde{\mathcal{M}}(X)=\sum_{p=1}^{n_{g}} z_{p} \Phi_{p}(X)
$$

where $z_{p}$ are the coefficients of the polynomial metamodel, more or less complicated to estimate, as discussed further.

\subsection{Probability Density Functions (PDFs) of the quantities of interest}

In numerous studies using SFE methods, only the mean value of the structural quantities of interest, several statistical moments, or a direct probability of failure are usually computed for a given problem [8]. These can be obtained with various techniques, through sensitivity or reliability approaches. We are interested in the full probabilistic characterization of the r.v. $Z=\mathcal{M}(X)$, i.e. we seek to approximate the PDF $p_{Z}$, as well as the mean $\mu_{Z}$, and standard deviation $\sigma_{Z}$. The $\operatorname{PDF} p_{\tilde{Z}}$ of $\tilde{Z}$ is a good approximation of $p_{Z}$ $\left(p_{\tilde{Z}}\right.$ allows to obtain quantities such as $\mu_{\tilde{Z}}$ and $\left.\sigma_{\tilde{Z}}\right)$. 
For the sake of simplicity, it is assumed in Eq. (4) that the mechanical system depends on one real r.v. $X$ whose law admits a PDF $p_{X}$ on the interval $I$. It is assumed that there exists a set of $n$ duals of intervals $\left(\Theta_{i}^{1}, \Theta_{i}^{2}\right)$ of $\mathbb{R}$ and bijections $\mathcal{M}_{i}: \Theta_{i}^{1} \rightarrow \Theta_{i}^{2}$ such that $\forall x \in \Theta_{i}^{1}, \mathcal{M}_{i}(x)=\mathcal{M}(x)$. Each function $\mathcal{M}_{i}$ admits a reciprocal $\mathcal{M}_{i}^{-1}$. Using a change of variables, $p_{Z}$ writes :

$$
p_{Z}(z)=\sum_{i \in I_{z}} p_{X}\left(\mathcal{M}_{i}^{-1}(z)\right)\left|\mathcal{M}_{i}^{-1 \prime}(z)\right|
$$

where $\mathcal{M}_{i}^{-1 \prime}$ is the derivative of the function $\mathcal{M}_{i}^{-1}$, and $I_{z}=\left\{i \in[1, n], z \in \Theta_{i}^{2}\right\}$. The same hypotheses are made for $\tilde{\mathcal{M}}$ with similar notations (in particular, the functions $\tilde{\mathcal{M}}_{i}$ are explicitly known if $\tilde{\mathcal{M}}$ is a polynomial function) to obtain the $\operatorname{PDF} p_{\tilde{Z}}$ of $\tilde{Z}$.

However, on typical mechanical problems under uncertainties, the quantities of interest depend on several random parameters. This approach can be extended to this case with similar hypotheses [19], making it suitable for a much larger range of mechanical problems. In this case, the PDF of $Z$ and its approximation $\tilde{Z}$ write in an expression similar to Eq. (4) :

$$
p_{Z}(z)=\sum_{i \in I_{z}} p_{X}\left(\mathcal{M}_{i}^{-1}(z)\right)\left|\operatorname{det}\left(\mathbf{D} \mathcal{M}_{i}^{-1}(z)\right)\right| \simeq p_{\tilde{Z}}(z)=\sum_{i \in I_{\tilde{z}}} p_{X}\left(\tilde{\mathcal{M}}_{i}^{-1}(z)\right)\left|\operatorname{det}\left(\mathbf{D} \tilde{\mathcal{M}}_{i}^{-1}(z)\right)\right|
$$

where $\mathbf{D} \mathcal{M}_{i}^{-1}$ is the Jacobian matrix of the function $\mathcal{M}_{i}^{-1}$, and $I_{\tilde{z}}=\left\{i \in[1, n], z \in \Theta_{i}^{2}\right\}$.

\section{Formulation of the proposed approach}

In this section, an approach is proposed to solve the previous problem, and to obtain the probabilistic estimation of the quantities of interest:

- A coupling between an adaptative condensation method and SFE approaches is introduced: the adaptive condensed SFEM. This method can be applied with different SFE approaches to provide the coefficients for the approximations of the mechanical response functions (Eq. (3)).

- The particular case of the coupling with a stochastic collocation method is presented.

- A semi-analytical approach to compute the PDF of the quantities of interest is introduced. This method uses the approximated response function to build the PDF of the quantities of interest $Z$.

\subsection{Adaptive condensed SFE method}

In the general framework of SFE methods, deterministic problems have to be solved to provide the coefficients $z_{p}$ for the approximation of $\tilde{\mathcal{M}}$ in the form of Eq. (3). The proposed approach allows to compute these coefficients efficiently. 


\subsubsection{Problem decomposition and static condensation}

For the sake of clarity, dependency on $y$ is implicitly considered in the next two paragraphs. To solve the nonlinear structural problem (Eq. (1)) defined on $\Omega, n_{I}$ subproblems $\Omega_{i}$ are considered. They are divided in two groups:

- $\Omega_{E}$ contains the $n_{E}$ elastic areas $\Omega_{i}$ numbered by $i \in E$.

- $\Omega_{N}$ contains the $n_{N}$ nonlinear areas (or areas of interest) $\Omega_{i}$ numbered by $i \in N$.

These groups are defined for any time $\tau$ such that $\Omega=\Omega_{Z} \cup \Omega_{N}$ (and $n_{I}=n_{E}+n_{N}$ ). The subdomains are such that areas interact together only by their geometrical boundaries (continuous media). The associated stiffness matrices of $\Omega_{E}$ and $\Omega_{N}$ can be obtained by assembling the stiffnesses of the areas $\Omega_{i}$. The global problem then writes :

$$
\left(\begin{array}{cc}
K_{E, E} & K_{E, N} \\
{ }^{t} K_{E, N} & K_{N, N}^{\tau}\left(u^{\tau}\right)
\end{array}\right) \cdot\left(\begin{array}{c}
u_{E}^{\tau} \\
u_{N}^{\tau}
\end{array}\right)=\left(\begin{array}{c}
F_{E}^{\tau} \\
F_{N}^{\tau}
\end{array}\right)
$$

Guyan's technique [15] applied to $\Omega_{E}$ allows to reduce this system by condensing it on the nonlinear subdomain $\Omega_{N}$. It follows the condensed stiffness matrix $\hat{K}$ and loading vector $\hat{F}$ :

$$
\begin{aligned}
& \hat{K}^{\tau}\left(u_{N}^{\tau}\right)=K_{N, N}^{\tau}\left(u_{N}^{\tau}\right)-{ }^{t} K_{E, N} K_{E, E}^{-1} K_{E, N} \\
& \hat{F}^{\tau}=F_{N}^{\tau}-{ }^{t} K_{E, N} K_{E, E}^{-1} F_{E}^{\tau}
\end{aligned}
$$

The condensed nonlinear system at time $\tau$ is finally obtained :

$$
\hat{K}^{\tau}\left(u_{N}^{\tau}\right) \cdot u_{N}^{\tau}=\hat{F}^{\tau}
$$

This system is reduced and contains all the information on the nonlinear behaviour. The solution $u_{E}^{\tau}$ on $\Omega_{E}$ can still be deduced by linear resolution (much less time-consuming) from the condensed solution $u_{N}^{\tau}$, using Eq. (6) :

$$
u_{E}^{\tau}=K_{E, E}^{-1}\left(F_{E}^{\tau}-K_{E, N} u_{N}^{\tau}\right)
$$

\subsubsection{Adaptive condensation}

The adaptive condensation method constitutes an upgrade of Guyan's method in two aspects [16] :

- It uses time-dependent decomposition in elastic areas (EA) and nonlinear areas (NLA): EA are promoted to NLA when they enter a nonlinear phase. Thus, it follows the evolution of the system in nonlinear behaviour. Therefore, $\Omega_{E}$ and $\Omega_{N}$ become respectively $\Omega_{E}^{\tau}$ and $\Omega_{N}^{\tau}$.

- It uses two levels of condensation : first on each condensed area, and second on the assembled condensed area $\Omega_{E}^{\tau}$. This optimizes the computation of stiffness matrices. 


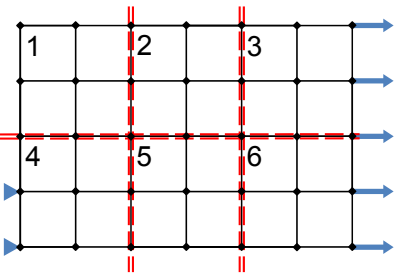

(a) Full problem (6 areas)

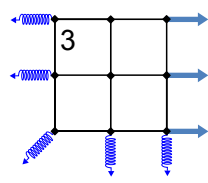

(b) Problem at time $\tau_{0}$

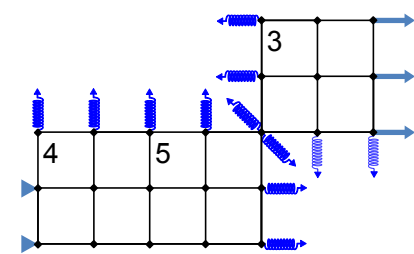

(c) Problem at time $\tau_{1}>\tau_{0}$

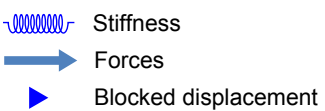

(d)

Figure 1: Illustration of a nonlinear mechanical problem solved with adaptive condensation [16]: the method eliminates the linear areas and adapts to the evolution of the system. At time $\tau_{0}$, only one area is nonlinear (\#3). At time $\tau_{1}$, three areas are nonlinear $(\# 3, \# 4, \# 5)$.

Computing the condensed stiffness matrix $\hat{K}^{\tau}$ is a time-consuming operation, mainly because of the large dimension of the inverse term $K_{E, E}^{-1}$ of Eq. (7). In practical cases, nonlinear behaviour appears in the initially elastic areas, and requires updates of the subdomains $\Omega_{E}^{\tau}$ and $\Omega_{N}^{\tau}$, and of the stiffness matrices $K_{E, E}$ and $\hat{K}^{\tau}$ (Fig. 1). Therefore, to limit computational cost in updates of $K_{E, E}^{-1}$, a second level of condensation is applied inside each area $\Omega_{i}$ on its boundaries, computing its equivalent stiffness $\hat{K}_{i, i}$ before starting the nonlinear simulation. The reduced stiffness matrices of each condensed area writes, for $j \in E, \hat{K}_{j, j}$. The global stiffness of condensed areas used in Eq. (6) is then assembled from condensed area stiffnesses :

$$
K_{E, E}=\left(\begin{array}{ccc}
\hat{K}_{1,1} & \ldots & \hat{K}_{1, n_{E}} \\
\vdots & \ddots & \vdots \\
\hat{K}_{n_{E}, 1} & \ldots & \hat{K}_{n_{E}, n_{E}}
\end{array}\right)
$$

The dimension of the matrix $K_{E, E}$ is reduced, thus reducing the computational cost of its inversion. The time-consuming operation is now to compute the reduced stiffnesses $\hat{K}_{j, j}$ of condensed areas. These stiffnesses are not updated through a deterministic simulation. Guyan's formula can be used to solve the system in each condensed area $\Omega_{j}$ and obtain the solution $u_{j}^{\tau}$. This is required to monitor the mechanical state of the system and update the decomposition if necessary.

It is worth noting that the inverses of the stiffness matrices are only used in the theoretical formulation, while the actual algorithms more often use matrix decomposition methods such as Cholesky or LU factorization $[20,21]$. However, the stiffness matrices are invertible when using a dualization method for boundary conditions (such as the double Lagrange multipliers used in Cast3M [17, 22]).

\subsubsection{Approximation of condensed quantities}

In SFE methods, each individual deterministic problem can be solved using finite elements with the adaptive condensation method described in 3.1.2. The adaptive condensation method reduces the cost of the FE simulation of the problem, but requires computing additional quantities. 
- The condensed stiffness matrices $\hat{K}_{i, i}$. These are pre-computed before the start of each nonlinear simulation.

- The condensed loading $\hat{F}_{i}^{\tau}$. These are computed throughout each nonlinear simulation.

- The local solution $u_{C i}^{\tau}$ inside each condensed area, computed regularly to monitor the mechanical state of the condensed areas (and trigger the promotion from EA to NLA [16]).

These quantities, which depend on the values of the random parameters, would have to be computed independently for any realization and nonlinear simulation run in the SFE framework. As this is a rather costly operation, this approach should be optimized. The proposed method therefore builds for the condensed stiffness of each area $\Omega_{i}$ a metamodel of order $\left(n_{K i}-1\right)$ on values of an appropriate subvector $X_{K i}$ of $X$. In each deterministic simulation, the necessary quantities are approximated using this metamodel. The reduced stiffness matrix is approximated at the beginning of the numerical simulation :

$$
\hat{K}_{i}(X) \simeq \sum_{p=1}^{n_{K i}} \hat{K}_{i}^{p} \Phi_{p}\left(X_{K i}\right)
$$

Following the same form, the condensed force vector $\hat{F}_{i}^{\tau}(X)$ of the condensed area $\Omega_{i}$ can also be written as :

$$
\begin{aligned}
& \hat{F}_{i}^{\tau}(X)=F_{M i}^{\tau}(X)-{ }^{t} K_{C i, M i}(X) K_{C i, C i}^{-1}(X) F_{C i}^{\tau}(X) \\
& \hat{F}_{i}^{\tau}(X) \simeq \sum_{p=1}^{n_{F i}} \hat{F}_{i}^{\tau, p} \Phi_{p}^{\prime}\left(X_{F i}\right)
\end{aligned}
$$

The solution $u_{C i}^{\tau}(X)$ on the eliminated degrees of freedom (DOFs) on area $\Omega_{i}$ also writes :

$$
\begin{aligned}
& u_{C i}^{\tau}(X)=K_{C i, C i}^{-1}(X)\left(F_{C i}^{\tau}(X)-K_{C i, M i}(X) u_{M i}^{\tau}(X)\right) \\
& u_{C i}^{\tau}(X) \simeq \sum_{p=1}^{n_{u i}} u_{C i}^{\tau, p} \Phi_{p}^{\prime \prime}\left(X_{u i}\right)
\end{aligned}
$$

where $\Phi_{p}, \Phi_{p}^{\prime}$, and $\Phi_{p}^{\prime \prime}$ are three bases of orthogonal polynomials, and $\hat{K}^{p}, \hat{F}^{p}$, and $u^{p}$ are the coefficients of the approximation.

It is worth noting that these metamodels are independent from the approximation of Eq (3), and can therefore be built using a different approach: for instance, the quantities necessary for condensation could be approximated by Taylor expansion, while the mechanical response could be approximated using a collocationbased SFE approach. This reduces the dimension of the pre-simulation polynomial metamodel computation.

\subsection{Condensed stochastic collocation}

Fig. 2 presents the general scheme of the condensed stochastic collocation approach. 


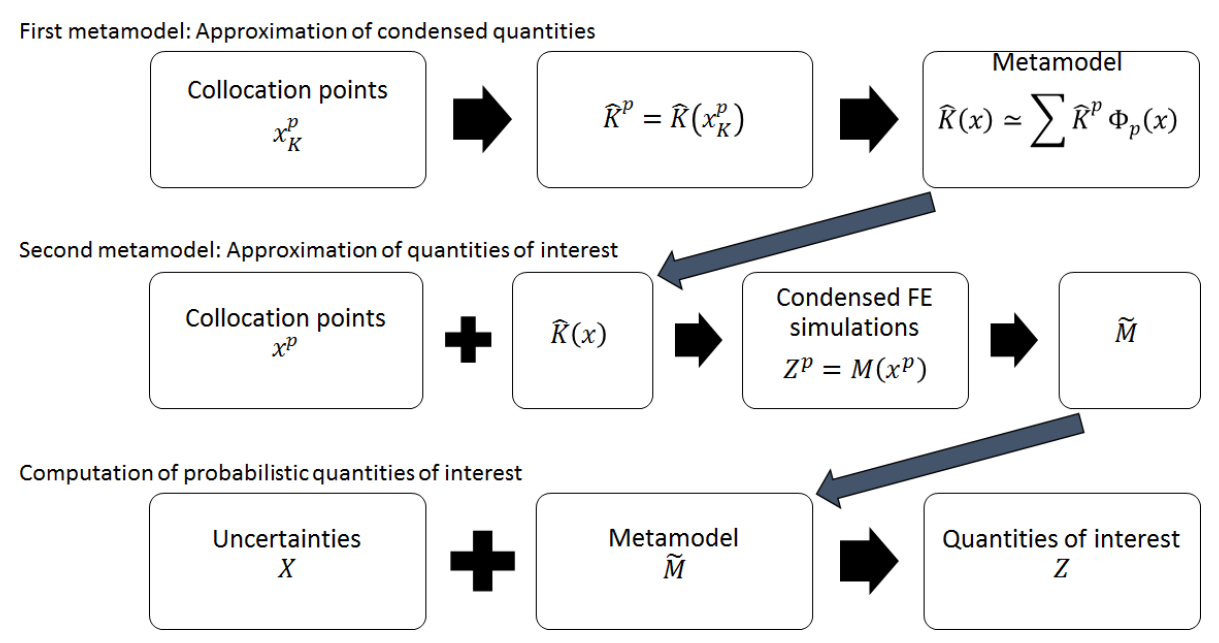

Figure 2: General scheme of the "Condensed Stochastic Collocation" approach.

\subsubsection{Stochastic collocation}

Stochastic collocation is a class of SFE methods in which the coefficients $z_{p}$ of the polynomial metamodel $\tilde{\mathcal{M}}$ of the mechanical response function $\mathcal{M}$ are computed using successive deterministic simulations on sample realizations. This study more specifically uses the Lagrange-polynomial based collocation method described in [23]. Given that the order of approximation is in general relatively low, Lagrange polynomials are used as basis of $\Phi_{p}$ in Eq. (3), instead of Hermite polynomials. The resulting stochastic collocation method solves a number of deterministic problems defined by the $n_{g}$ collocation points $z^{p}=\mathcal{M}\left(x^{p}\right)$ of the response function $\tilde{\mathcal{M}}$ defined in Eq. (3). The collocation points $x^{p}$ are chosen accordingly to the Gauss-Hermite quadrature, to minimize the Runge oscillation phenomenon of Lagrange polynomials [20].

\subsubsection{Adaptive condensed stochastic collocation}

The proposed condensed stochastic collocation method is the application of coupling between the stochastic collocation method and the adaptive condensation method. It is the specific form of the adaptive condensed SFEM in a collocation-based framework. The proposed approach is actually a double-collocation based approach:

- A first collocation-based metamodel is built to approximate the condensation quantities (stiffness matrices) depending on the problem's random parameters.

- A second metamodel is built for the mechanical response function: this requires computation of deterministic simulations on collocation points.

- The mechanical function on collocation points is computed using the adaptive condensation method. The necessary condensation quantities are approximated in these simulations using the first metamodel. 
In the particular form of condensed stochastic collocation, the approximated random condensed stiffness matrices $\hat{K}_{i}(X)$, random condensed force vectors $\hat{F}_{i}^{\tau}(X)$ and random displacement fields $u_{C i}^{\tau}(X)$ in the condensed areas are approximated based on a collocation metamodel. At first, for each area $i$, the random variables of $X$ that influence the condensed quantities of area $i$ (in particular, the reduced stiffness), are determined and ordered in a subvector $X_{K i}$.

The choice of the subvectors $X_{K i}$ and of the order of approximation $\left(n_{K}-1\right)$ depends on the nature of the uncertainties taken into account

- Random variable that influence the elastic stiffness (in particular, elastic material properties such as Young's modulus or Poisson coefficient) should be included in the subvector $X_{K i}$. The stiffness matrices are approximated linearly on those variables (therefore $n_{K}=2$ ) in the proposed applications.

- Random variables that have no impact on the elastic stiffness (such as loading or elastic limit) are not considered in the vector $X_{K i}$.

- Random variables that influence nonlinearly the stiffnesses (such as position of inclusions) are included in the vector $X_{K i}$, with an order of approximation $n_{K} \geq 3$.

Obviously the choice of the rvs to be included in the local metamodels is largely case-dependant. For instance, on civil engineering applications, concrete properties are often represented by uncertainty on local model parameters, such as Young's modulus or damage threshold. These properties are often considered to vary independently between the different lifts. Therefore, for a given area $i$, a good strategy is to take into account in $X_{K i}$ only the elastic modulus of the lift in which the area is situated.

On these subvectors $X_{K i}$, collocation points $x_{K i}^{p}$ are determined accordingly to the Gauss-Hermite quadrature (as for the first metamodel), on which the values $\hat{K}_{i}^{p}$ of the condensed stiffnesses are calculated. Note that the stiffness matrices $\left(\hat{K}_{i}^{p}, K_{C i, M i}^{p}, K_{C i, C i}^{p}\right)$ are calculated at these points, but also the (time-consuming) inverse terms $\left(\left(K^{-1}\right)^{p}\right)$ that will allow to compute other condensed quantities.

A Lagrange-polynomial based metamodel is then built by collocation on those points, for the estimation of the condensed stiffnesses at the start of each condensed nonlinear simulation:

$$
\hat{K}_{i}(X) \simeq \sum_{p=1}^{n_{K}} \hat{K}_{i}^{p} \Phi_{p}\left(X_{K i}\right)
$$

where $\Phi_{p}$ are the Lagrange polynomials defined on the $n_{K}$ points of the metamodel, $X_{K i}$ is the selected sub-vector of $X$, and $\hat{K}^{p}$ are the values at the collocation points $x_{K i}^{p}$.

During the condensed nonlinear simulations, it is necessary to compute the reduced loading transmitted by the condensed areas to the areas of interest. It is also necessary to compute regularly the displacements and promotion criteria in the condensed areas. In the condensed stochastic collocation, these quantities are 
first computed using the collocation values of the inverted stiffnesses $\left(K^{-1}\right)^{p}$ :

$$
\begin{aligned}
\hat{F}_{i}^{\tau, p}(X) & \simeq F_{M i}^{\tau}(X)-\left({ }^{t} K_{C i, M i}\left(K_{C i, C i}^{-1}\right)^{p}\right) F_{C i}^{\tau}(X) \\
u_{i}^{\tau, p}(X) & \simeq\left(K_{C i, C i}^{-1}\right)^{p}\left(F_{C i}^{\tau}(X)-K_{C i, M i} u_{M i}^{\tau}(X)\right)
\end{aligned}
$$

Afterwards, the condensed quantities are approximated from these values using a Lagrange polynomial metamodel, and used within the nonlinear simulation:

$$
\begin{aligned}
\hat{F}_{i}^{\tau}(X) & \simeq \sum_{p=1}^{n_{K}} \hat{F}_{i}^{\tau, p}(X) \Phi_{p}\left(X_{K i}\right) \\
u_{C i}^{\tau}(X) & \simeq \sum_{p=1}^{n_{K}} u_{C i}^{\tau, p}(X) \Phi_{p}\left(X_{K i}\right)
\end{aligned}
$$

One of the advantages of this approach is that it allows to use the same choice of r.v.s $X_{K i}$ and collocation points $x_{K i}^{p}$, and therefore a single metamodel for all condensation quantities.

It can be used with almost no user input, for instance by simply taking all variables into account in the subvector $\left(X_{K i}=X\right)$, with a reduced order of approximation (typically $n_{K}=2$ ). Since the number of necessary computations of the reduced stiffnesses (collocation values) grows in $\left(n_{K}\right)^{n}$ (where $n$ is the total number of rvs), it is largely reduced in this way if the number of rvs is high. However, with some user expertise to identify the influence of the different rvs on the local stiffnesses, it can reduce more drastically the number of necessary calculations of reduced stiffnesses. On a typical civil engineering study, a single computation of the reduced stiffnesses may require several minutes on a dedicated simulation node.

\subsection{Approximation of the PDF of quantities of interest}

The objective is to obtain an approximation of the probabilistic characterization of the quantities of interest $Z$. The approximation $\tilde{Z}$ of $Z$ is available from SFE methods and has then to be characterized (in particular, its PDF $p_{\tilde{Z}}$ must be computed). Two different approaches are presented for this purpose, that will be used and compared in the following parts.

\subsubsection{Monte-Carlo estimation}

The first method available consists in applying the Monte-Carlo method on the response function. This approach is proposed in [23], with a polynomial metamodel built by collocation. For a given (arbitrarily large) sample $\left(x^{1}, \ldots, x^{N}\right)$ of realizations of $X$, the values of the approximated response function $\left(\tilde{\mathcal{M}}\left(x^{1}\right), \ldots, \tilde{\mathcal{M}}\left(x^{N}\right)\right)$ are computed. The experimental distribution can then be statistically analyzed. This technique presents numerous advantages, mainly a simplicity of implementation and robustness, and a limited computational load, as the polynomial response surface requires very little calculation. Also, it matches the objective of building a full probabilistic characterization (with PDF) of the approximated quantities of interest $\tilde{Z}$. However, it still has drawbacks, such as a low rate of convergence and the necessity to post-process large quantities of data. 


\subsubsection{Semi-analytical approach}

In several cases, where the form of the metamodel $\tilde{\mathcal{M}}$ is rather simple, another approach is proposed here to avoid these disadvantages. The proposed technique, called semi-analytical approach, uses the expression of the metamodel $\tilde{\mathcal{M}}$ to compute directly the PDF of the approximated quantities of interest $\tilde{Z}$. It offers, under certain hypotheses, an alternative to Monte-Carlo type methods, or other kinds of numerical methods applied on the metamodel, and uses its polynomial nature. In the cases where the hypotheses are not matched, or hard to obtain, applying the Monte-Carlo approach to the polynomial expansion may still be required. Using either Eq.(4) or Eq.(5) with the approximation $\tilde{\mathcal{M}}$ of the mechanical response allows to obtain the PDF of $\tilde{Z}$.

For the sake of clarity, we focus at first on the particular case of a single r.v. Considering a standard Gaussian PDF, and assuming that $\mathcal{M}^{\prime}$ is non-null at $x=\tilde{\mathcal{M}}_{i}^{-1}(z)$, the PDF of $\tilde{Z}$ is deduced (Eq.(4)) :

$$
p_{\tilde{Z}}(z)=\sum_{i \in I_{\tilde{z}}} \frac{1}{\sqrt{2 \pi}} \exp \left(-\frac{1}{2}\left(\tilde{\mathcal{M}}_{i}^{-1}(z)\right)^{2}\right)\left|\frac{1}{\tilde{\mathcal{M}}^{\prime}\left(\tilde{\mathcal{M}}_{i}^{-1}(z)\right)}\right|
$$

Considering the polynomial nature of $\tilde{\mathcal{M}}$, its derivative $\tilde{\mathcal{M}}^{\prime}$ can be explicitly computed, and the intervals $I_{z}$ where $\tilde{\mathcal{M}}$ is bijective are given by the roots of $\tilde{\mathcal{M}}^{\prime}$. The local reciprocals $\tilde{\mathcal{M}}_{i}^{-1}$ of $\tilde{\mathcal{M}}$ may be obtained directly if the order of the expansion is low: in the cases where $N_{s c} \in[2,3]$, explicit solutions exist to the equation $\tilde{\mathcal{M}}(x)=z$. In the other cases, where the metamodel is of higher degree, local approximations of the reciprocals $\tilde{\mathcal{M}}_{i}^{-1}$ may be used.

An approach is to compute the values $\tilde{\mathcal{M}}_{i}\left(x^{j}\right)=\tilde{z}^{j}$ of the function $\tilde{\mathcal{M}}_{i}$ on an arbitrary number of points $x^{j} \in \Theta_{i}^{1}$. On each point $x^{j}$, the first and second derivatives $\tilde{\mathcal{M}}_{i}^{\prime}\left(x^{j}\right)$ and $\tilde{\mathcal{M}}_{i}^{\prime \prime}\left(x^{j}\right)$ can be computed, either directly (if the expression of $\tilde{\mathcal{M}}$ is explicitly known), or using a finite-difference scheme with $\tilde{\mathcal{M}}_{i}\left(x^{j-1}\right)=\tilde{z}^{j-1}$ and $\tilde{\mathcal{M}}_{i}\left(x^{j+1}\right)=\tilde{z}^{j+1}$. These derivatives also provide the values of derivatives of the reciprocal $\tilde{\mathcal{M}}_{i}^{-1 \prime}\left(\tilde{z}^{j}\right)$ and $\tilde{\mathcal{M}}_{i}^{-1 \prime}\left(\tilde{z}^{j}\right)$. Therefore, a degree-2 Taylor approximation of $\tilde{\mathcal{M}}_{i}^{-1}$ around $z^{j}$ can be built. This approximation is to be used in Eq. (22) to compute values of the PDF of the quantity of interest $p_{\tilde{Z}}$.

In the general case however, the response function and its approximation $\tilde{\mathcal{M}}$ are functions of $k$ scalar r.v.s that provide $k^{\prime}$ scalar values: $\tilde{\mathcal{M}}: \Theta^{1} \subset \mathbb{R}^{k} \rightarrow \Theta^{2} \subset \mathbb{R}^{k^{\prime}}$ with, $\forall x=\left(x_{1}, \ldots, x_{k}\right) \in \Theta^{1}, \tilde{\mathcal{M}}(x)=$ $\left(\tilde{\mathcal{M}}_{1}(x), \ldots, \tilde{\mathcal{M}}_{k^{\prime}}(x)\right)$. In some cases, local bijections $\tilde{\mathcal{M}}_{i}$ can be built. A first step is to use $k$-dimensional sets as $\Theta^{1}$ and $\Theta^{2}$.

- If $k^{\prime}>k$, a possible approach is to separate the values in multiple response functions. The single response function $\tilde{\mathcal{M}}: \mathbb{R}^{k} \rightarrow \mathbb{R}^{k^{\prime}}$ is replaced by a serial of functions $\mathcal{A}, \mathcal{B}, \mathcal{C} \ldots: \mathbb{R}^{k} \rightarrow \mathbb{R}^{k}$ treated as separate responses.

- If $k^{\prime}<k$, a possible approach is to build a response function $\mathcal{N}: \mathbb{R}^{k} \rightarrow \mathbb{R}^{k}$ such that $\mathcal{N}(x)=$ $\left(\tilde{\mathcal{M}}_{1}(x), \ldots, \tilde{\mathcal{M}}_{k^{\prime}}(x), x_{k^{\prime}+1}, \ldots, x_{k}\right)$. 
A combination of both methods can be necessary to obtain $k$-dimensional sets in some cases. The series of sets $\left\{\Theta_{1}^{1}, \ldots, \Theta_{k}^{1}\right\}$ and $\left\{\Theta_{1}^{2}, \ldots, \Theta_{k}^{2}\right\}$ and the local bijections $\mathcal{N}_{i}$ can then be built. Their reciprocals $\mathcal{N}_{i}^{-1}$ may be built explicitly in some cases. However, in the general case, they will be approximated using methods similar to the 1-dimensional case such as Taylor expansion. The PDF of $\mathcal{N}(X)$ can be obtained using Eq. (5) with the functions $\mathcal{N}_{i}$. The PDF of $\tilde{Z}=\tilde{\mathcal{M}}(X)$ can then be deduced using conditional probability.

Therefore, this can be considered as a semi-analytical method to obtain an approximation of the response of the probabilistic problem. This method requires to extract the metamodel, define the sets where it is bijective and compute the derivatives. In particular it requires a mathematical study to obtain the bijectivity of a response function in the multivariate case. This may represent a large amount of work for high-order metamodels with a large number of random variables. However, for problems with low numbers of random parameters, the proposed semi-analytical approach provides analytical-quality results.

\section{Application: Steel bar with localized defect}

\subsection{Problem description}

This test case aims at illustrating the principle and applicability of the proposed method on a small twodimensional, locally nonlinear problem: in particular, it can be simplified to be solved analytically. it also includes an arbitrarily small area with nonlinear behaviour. The results are compared to a state-of-the-art SFE method $[23,24]$ and an analytical solution. The structure is a steel bar with a central defect, undergoing tensile loading. The objective is to build a metamodel of the mechanical behaviour and to extract a PDF for the maximum plastic strain $\varepsilon_{p}$ at the end of the loading.

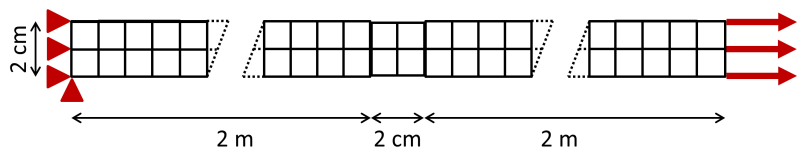

Figure 3: Scheme of the steel bar with boundary conditions

The bar is modeled in two dimensions under the plane stress hypothesis. Fig. 3 presents the scheme of the bar. The bar is meshed uniformly with square bilinear elements (QUA4). The central defect is modeled as a $2 \mathrm{~cm}$ long segment. At this position, the height of the bar is reduced by $5 \%$. The relation between both end parts of the bar and the central part is ensured by kinematic relations on nodal displacements. Fig. 4 presents the meshes (central part): different mesh size will be used to evaluate scalability. Rigid body motion is blocked. The loading is an horizontal force applied at the right end, to create uniaxial loading conditions. It yields a stress of $475 \mathrm{MPa}$ in both lateral parts, and $f=500 \mathrm{MPa}$ in the central part.

The steel is modeled using Von Mises' plasticity model with linear hardening. Parameters used in the model are typical values from European construction codes [25], given in Table 1. All material parameters are considered homogeneous in the bar. 


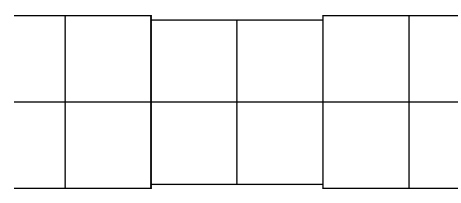

(a) Mesh size $10 \mathrm{~mm}$

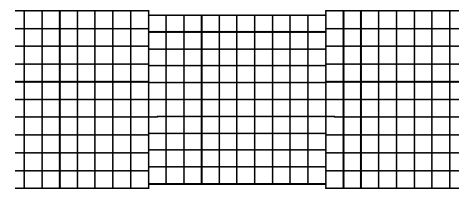

(b) Mesh size $2 \mathrm{~mm}$

Figure 4: Two different meshes of the bar

Table 1: Material parameters for the steel mechanical model

\begin{tabular}{|c|c|c|c|c|c|}
\hline \multirow{2}{*}{ Young's modulus } & \multirow{2}{*}{ Poisson ratio } & \multicolumn{2}{|c|}{ Yield strain* } & \multirow{2}{*}{ Ultimate strain } & \multirow{2}{*}{ Ultimate stress } \\
\cline { 3 - 5 } & & Mean & Std. dev. & \\
\hline$E$ & $\nu$ & $\varepsilon_{y}^{\mu}$ & $\varepsilon_{y}^{\sigma}$ & $\varepsilon_{u}$ & $f_{u}$ \\
\hline $210 \mathrm{GPa}$ & 0.28 & $2.38 \times 10^{-3}$ & $2.38 \times 10^{-5}$ & 0.05 & $540 \mathrm{MPa}$ \\
\hline
\end{tabular}

To model the uncertainty on the mechanical properties of the steel, a random variable is introduced to represent the yield strain $\varepsilon_{y}$. Its distribution follows a truncated Gaussian law, with mean $\varepsilon_{y}^{\mu}=2.38 \times 10^{-3}$ and standard deviation $\varepsilon_{y}^{\sigma}=2.38 \times 10^{-5}$ ( $\operatorname{such}$ that $f=E \times \varepsilon_{y}^{\mu}$ ). The normalized distribution of $X$ is truncated on $[-8,8]$ (8 standard deviations from average). The truncated standard law avoids the creation of nonphysical values of $\varepsilon_{y}$ to be created, since it eliminates values higher than $f_{u} / E$, and negative values. The choice of the truncated standard distribution has been made to simplify the analytical study, as it presents a simple PDF, is symmetric, and allows a collocation point to be placed at $X=0$. This truncation introduces a error in the estimation of the PDF. However, this error is estimated to be lower than $10^{-13}$, and is therefore neglected.

The elastic properties and ultimate strain and stress do not depend on the value of the random yield strain $\varepsilon_{y}$, but the hardening modulus and yield stress do. Fig. 5 presents the stress-strain evolutions of the model for three different values of $\varepsilon_{y}$.

\subsection{Analytical study}

This test case can be analytically solved, by considering the central defect as a one-dimensional plasticity problem. Knowing that the reduced-section zone is plasticized, the plastic strain $\varepsilon_{p}$ can be computed depending on the yield limit $\varepsilon_{y}$ :

- if $0 \leq \varepsilon_{y} \leq \frac{f}{E}$

$$
\varepsilon_{p}=\left(\frac{E \varepsilon_{u}-f_{u}}{E}\right) \frac{f-E \varepsilon_{y}}{f_{u}-E \varepsilon_{y}}
$$

- if $\frac{f}{E} \leq \varepsilon_{y} \leq \frac{f_{u}}{E}$

$$
\varepsilon_{p}=0
$$




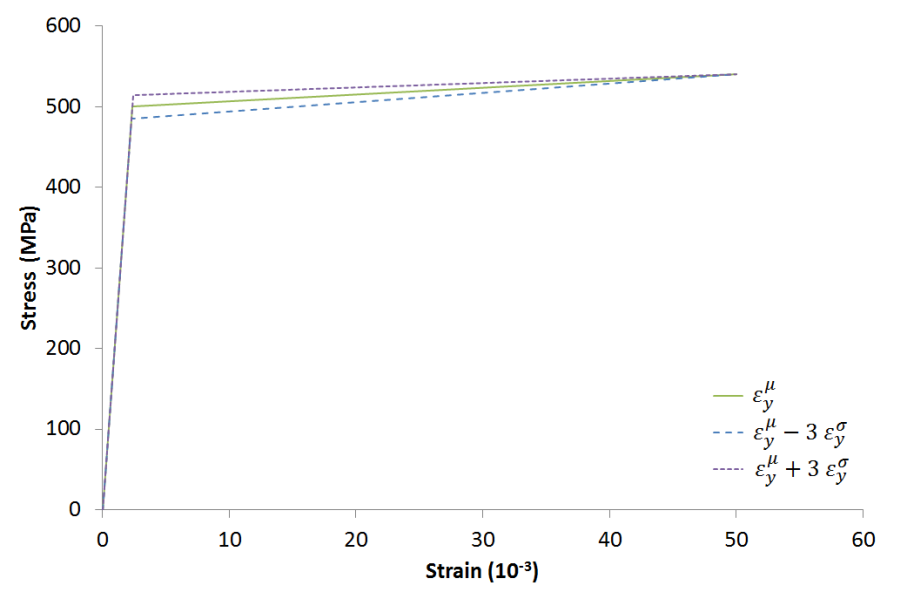

Figure 5: Stress-strain evolutions of the steel model for different values of $\varepsilon_{y}$.

- The other cases are not considered, due to physical considerations and model limitations (in particular, a negative yield limit or a yield limit greater than the ultimate limit).

The yield strain $\varepsilon_{y}$ of the steel bar depends on a standard r.v. $X$ :

$$
\varepsilon_{y}=\varepsilon_{y}^{\mu}+\varepsilon_{y}^{\sigma} X
$$

Therefore, the mechanical response (maximum plastic strain in the bar) can be directly written as a function of $x$, realization of $X$ :

- if $-\frac{\varepsilon_{y}^{\mu}}{\varepsilon_{y}^{\sigma}} \leq x \leq 0$

$$
\varepsilon_{p}(x)=\left(E \varepsilon_{u}-f_{u}\right) \frac{-\varepsilon_{y}^{\sigma} x}{\left(f_{u}-f\right)-E \varepsilon_{y}^{\sigma} x}
$$

- if $0 \leq x \leq \frac{f_{u}-f}{E \varepsilon_{y}^{\sigma}}$

$$
\varepsilon_{p}(x)=0
$$

- Other cases are not considered. Their probabilities are nullified due to the truncation of the probability law at \pm 8 standard deviations.

The plastic strain is null when $x \geq 0$ (yield strain greater than its average value). Therefore useless and costly numerical simulations are avoided by considering only the negative part of the distribution, where nonlinear mechanical behaviour actually appears $\left(\varepsilon_{p}(x)>0\right)$. From the mechanical response function (Eq. $26,27)$, the null value of the mechanical response corresponds to a finite probability :

$$
\mathcal{P}\left(\varepsilon_{p}=0\right)=\mathcal{P}(X \geq 0)=\frac{1}{2}
$$


The PDF $p_{p}$ of the r.v. which represents the plastic strain value $\varepsilon_{p}$ can be directly written, knowing the $\mathrm{PDF} p_{X}$ of the r.v. $X$ and the analytical mechanical response function $\varepsilon_{p}$, using Eq. (4):

$$
\left.\forall x \in] 0 ; \varepsilon_{u} \frac{f}{f_{u}}\right], p_{p}(x)=\frac{\left(\left(f_{u}-f\right)-\frac{E x\left(f_{u}-E \varepsilon_{y}^{\mu}\right)}{E x-\left(E \varepsilon_{u}-f_{u}\right)}\right)^{2}}{\sqrt{2 \pi} \varepsilon_{y}^{\sigma}\left(E \varepsilon_{u}-f_{u}\right)\left(f_{u}-f\right)} \exp \left(-\left[\frac{x\left(f_{u}-E \varepsilon_{y}^{\mu}\right)}{\sqrt{2} \varepsilon_{y}^{\sigma}\left(E x-\left(E \varepsilon_{u}-f_{u}\right)\right)}\right]^{2}\right)
$$

This PDF will serve as a reference result for the simulations in the following.

\subsection{Adaptive condensation and condensed collocation parameters}

On this test case, the adaptive condensation method [16] is applied on the simulations with 32 areas. Since the only random parameter applied is the yield strain, which has no impact on the stiffness, the order $\left(n_{K i}-1\right)$ of the metamodel for the condensed stiffness is chosen equal to zero: condensed stiffnesses are in fact independent on the r.v., and the displacements in the condensed zones are interpolated from only one value (i.e., directly computed) at each step :

$$
\begin{aligned}
\hat{K}_{i}(X) & \simeq \hat{K}_{i} \\
\hat{F}_{i}^{\tau}(X) & \simeq F_{M i}^{\tau}(X)-\left({ }^{t} K_{C i, M i} K_{C i, C i}{ }^{-1}\right) F_{C i}^{\tau}(X) \\
u_{C i}^{\tau}(X) & \simeq K_{C i, C i}{ }^{-1}\left(F_{C i}^{\tau}(X)-K_{C i, M i} u_{M i}^{\tau}(X)\right)
\end{aligned}
$$

As aforementioned, the metamodels are only built on and used for the negative part of the r.v's law. The choice of the collocation points is then modified: since the metamodel is used only for negative values of $X$, only the negative quadrature points are used in the polynomial expansion.

Considering a 3-point collocation, the metamodel $\tilde{\mathcal{M}}$ is a degree 2 polynomial. Its reciprocal $\tilde{\mathcal{M}}^{-1}$ can be directly computed using the delta method on the intervals where the polynomial is bijective. Its derivative $\tilde{\mathcal{M}}^{\prime}$ (degree 1 polynomial) can also be directly obtained. The PDF $p_{p}$ of the random plastic strain can then be obtained using Eq. (22).

\subsection{Results}

Fig. 6 presents the metamodels built with the "classical" and condensed stochastic collocation methods both using 3 collocation points. It also compares it to the analytical solution of Eqs. (26)-(27) and illustrates the choice of the collocation points. On the figure, both collocation metamodels are similar. They correspond to the analytical solution at the collocation points. It indicates that the simulation and the analytical solution are in good agreement. Far from the collocation points, the metamodels are less accurate. This implies that the collocation points and basis of the metamodel are to be chosen in accordance with the objectives of the study.

The results obtained by the two collocation methods are in good agreement with the analytical function. With both collocation methods $\left(N_{s c}=3\right)$, we obtain values of the quantity of interest $\varepsilon_{p}^{1}=0$, 


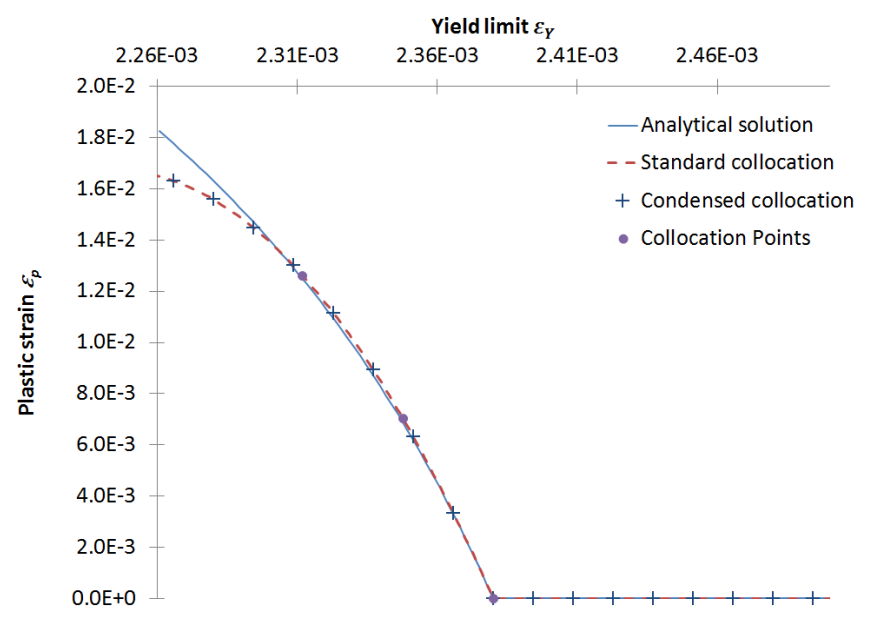

Figure 6: Analytical and collocated evolutions of plastic strain $\varepsilon_{p}$ depending on the r.v. $\varepsilon_{Y}$.

$\varepsilon_{p}^{2}=7.04 \times 10^{-3}, \varepsilon_{p}^{3}=1.26 \times 10^{-2}$ for the collocation points $x^{1}=0, x^{2}=-1.36, x^{3}=-2.86$. For positive values of the standardized r.v. $X$, values of the plastic strain $\varepsilon_{p}$ are taken null, as in the analytical study. The metamodel, computed by Lagrange polynomials, writes

$$
\tilde{\mathcal{M}}(X)=-5.22 \times 10^{-4} X^{2}-5.90 \times 10^{-3} X
$$

The metamodel $\tilde{\mathcal{M}}$ (degree 2 polynomial) reaches a maximum around a plastic strain of $1.67 \times 10^{-2}$. Therefore, the PDF of the plastic strain obtained with the metamodel is null for values higher than $1.67 \times 10^{-2}$. For values in $\left[0,1.67 \times 10^{-2}\right], \tilde{\mathcal{M}}$ admits two local reciprocal bijections $\tilde{\mathcal{M}}_{1}^{-1}:\left[0,1.67 \times 10^{-2}\right] \rightarrow[-5.66,0]$ and $\tilde{\mathcal{M}}_{2}^{-1}:\left[0,1.67 \times 10^{-2}\right] \rightarrow[-11.3,-5.66]$ (the case where $X<-11.3$ is not taken into account, since the distribution of $X$ is truncated on $[-8,8])$. Their expressions write:

$$
\begin{aligned}
& \tilde{\mathcal{M}}_{1}^{-1}\left(\mathcal{E}_{p}\right)=-959 \times\left(5.90 \times 10^{-3}-\sqrt{3.48 \times 10^{-5}-2.09 \times 10^{-3} \mathcal{E}_{p}}\right) \\
& \tilde{\mathcal{M}}_{2}^{-1}\left(\mathcal{E}_{p}\right)=-959 \times\left(5.90 \times 10^{-3}+\sqrt{3.48 \times 10^{-5}-2.09 \times 10^{-3} \mathcal{E}_{p}}\right)
\end{aligned}
$$

From these expressions we deduce the semi-analytical PDF for the r.v. $\mathcal{E}_{p}$ :

$$
p_{\tilde{p}}(z)=\frac{1}{\sqrt{2 \pi}}\left[\frac{\exp \left(-0.5\left(\tilde{\mathcal{M}}_{1}^{-1}(z)\right)^{2}\right)}{\left|\tilde{\mathcal{M}}^{\prime}\left(\tilde{\mathcal{M}}_{1}^{-1}(z)\right)\right|}+\frac{\exp \left(-0.5\left(\tilde{\mathcal{M}}_{2}^{-1}(z)\right)^{2}\right)}{\left|\tilde{\mathcal{M}}^{\prime}\left(\tilde{\mathcal{M}}_{2}^{-1}(z)\right)\right|}\right]
$$

Fig. 7 presents three PDFs $p_{p}$ for the plastic strain $\varepsilon_{p}$

- The analytical solution, obtained from Eq. (29) (1D analytical mechanical model);

- The semi-analytical PDF, obtained from Eq. (30); 
- The PDF obtained by standard stochastic collocation associated to a Monte-Carlo approach [23], from 25,000 calls to the metamodel of the condensed collocation $\left(N_{s c}=3\right)$.

The Monte-Carlo PDF is less accurate than the semi-analytical one. However it would eventually converge to the semi-analytical evolution using more realizations.

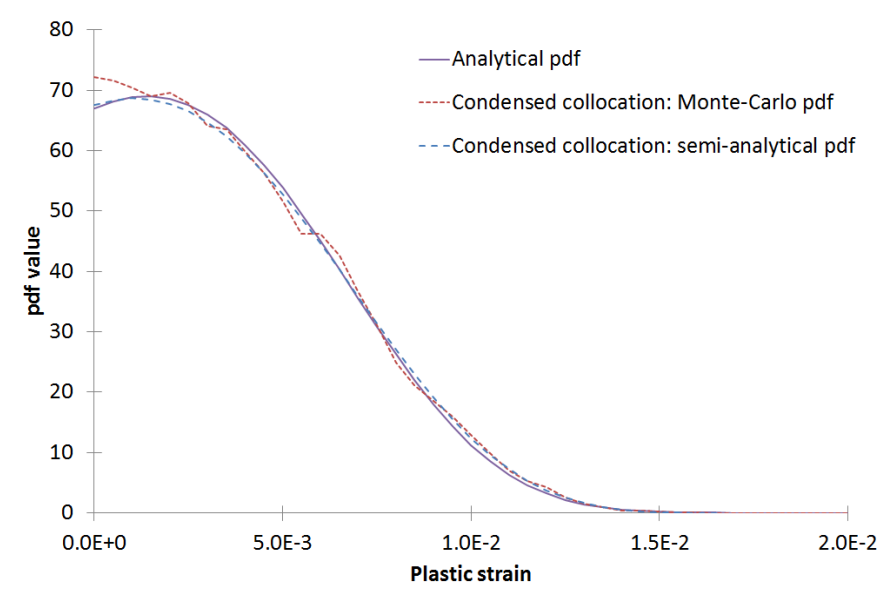

Figure 7: Analytical and collocated PDFs of the plastic strain

Table 2 presents the processor times necessary for both the standard and condensed stochastic collocation methods to compute metamodels of the mechanical problem with various mesh sizes. Presented metamodels are built with either 3 or 5 collocation points, and use therefore respectively degree 2 or degree 4 polynomials. The condensed collocation, which provides similar metamodels to the standard collocation (Fig. 6), requires less computation time on a given problem. When the problem becomes larger, for instance with mesh refinement, the gap becomes clearer. Consequently, for a given computation time, the condensed collocation method is able to improve the quality of the results.

Table 2: Processor times for two simulations of the steel bar, on a standard dual-core workstation. Processor times of the full Monte-Carlo calculations are estimated for 10,000 realizations, to give an order of magnitude.

\begin{tabular}{|l|l|l|l|l|}
\hline \multicolumn{2}{|c|}{ Simulation } & Stochastic coll. & Condensed coll. & Monte-Carlo (est.) \\
\hline $\begin{array}{l}10 \mathrm{~mm} \text { mesh } \\
(2,426 \text { DOFs })\end{array}$ & $3 \mathrm{pts}$ & $19.2 \mathrm{~s}$ & $17.6 \mathrm{~s}$ & $\sim 65 \times 10^{3} \mathrm{~s}$ \\
\cline { 2 - 4 } & $5 \mathrm{pts}$ & $32.8 \mathrm{~s}$ & $30.0 \mathrm{~s}$ & \\
\hline $\begin{array}{l}1 \mathrm{~mm} \text { mesh } \\
(168,924 \text { DOFs })\end{array}$ & $3 \mathrm{pts}$ & $1286 \mathrm{~s}$ & $254 \mathrm{~s}$ & $\sim 4.2 \times 10^{6} \mathrm{~s}$ \\
\cline { 2 - 4 } & $2110 \mathrm{~s}$ & $465 \mathrm{~s}$ & \\
\hline
\end{tabular}

Fig. 8 presents the speed-up values obtained by condensed collocation compared to stochastic collocation to calculate the metamodels as a function of the relative problem size. 3 and 5 point collocation are considered. Both results show a gradual increase of the speed-up, up to a plateau at around 5. Obviously 
the values of this speed-up are highly dependent on the problem configuration and decomposition. However similar trends can be expected in general.

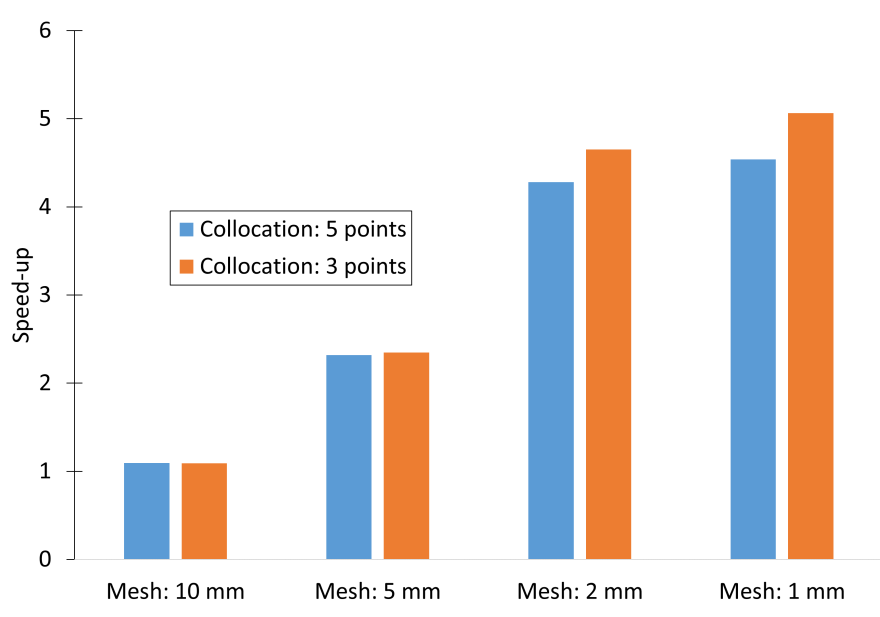

Figure 8: Speed-up of condensed collocation for different meshes.

\subsection{Effect of the stochastic dimension}

In order to evaluate the ability of the proposed method to scale to a higher stochastic dimension, a variant of this problem is studied, which contains additional random parameters. In this variant, 6 random variables affect the problem. They follow log-normal distributions, with the parameters given in Table 3 . The condensed stochastic collocation is used to build a polynomial metamodel using 3 collocation points on each variable. Building the metamodel with standard or condensed stochastic collocation will therefore require 729 collocation points (and 729 nonlinear simulations).

Table 3: Random parameters in the variant of the bar application.

\begin{tabular}{|l|c|c|c|c|c|c|}
\hline Parameter & Elastic modulus & Poisson ratio & Width & Yield limit & Ult. strain & Ult. stress \\
\hline Symbol & $E$ & $\nu$ & $L^{\prime}$ & $\varepsilon_{y}$ & $\varepsilon_{u}$ & $f_{u}$ \\
\hline Mean & $210 \mathrm{GPa}$ & 0.28 & 1.0 & $2.38 \times 10^{-3}$ & $5.0 \times 10^{-2}$ & $540 \mathrm{MPa}$ \\
\hline Std. dev. & $4.2 \mathrm{GPa}$ & $5.6 \times 10^{-3}$ & $5 \times 10^{-3}$ & $4.76 \times 10^{-5}$ & $2.0 \times 10^{-3}$ & $21.6 \mathrm{MPa}$ \\
\hline
\end{tabular}

However, only 3 of the random variables (Young's modulus, Poisson coefficient and bar width) influence the elastic properties of the bar. Since these quantities are expected to have linear impact on the local stiffness matrices, the order of approximation for the second polynomial expansion is chosen at $\left(n_{K}-1\right)=1$ : the stiffness estimation will use 2 collocation points on each variable. Hence, the fully coupled approach using the low-order expansion for the reduced stiffnesses allows the pre-processing to be considerably lightened. In this configuration, only 8 sets of reduced stiffnesses have to be effectively computed, while an uncoupled 
approach would require 729 calculations. Using a $10 \mathrm{~mm}$ mesh, this represents a pre-processing time of $7.0 \mathrm{~s}$ with the coupled approach, and $619.4 \mathrm{~s}$ without the second metamodel. Using a $1 \mathrm{~mm}$ mesh, the pre-processing time with the coupled approach reaches 370.3 s. Fig. 9 presents the PDF of the plastic strain $\varepsilon_{p}$ obtained by condensed collocation with the Monte-Carlo approach on this variant of the problem. In this configuration, the probability of a null value for the plastic strain is $\mathcal{P}\left(\varepsilon_{p}=0\right) \simeq 0.32$.

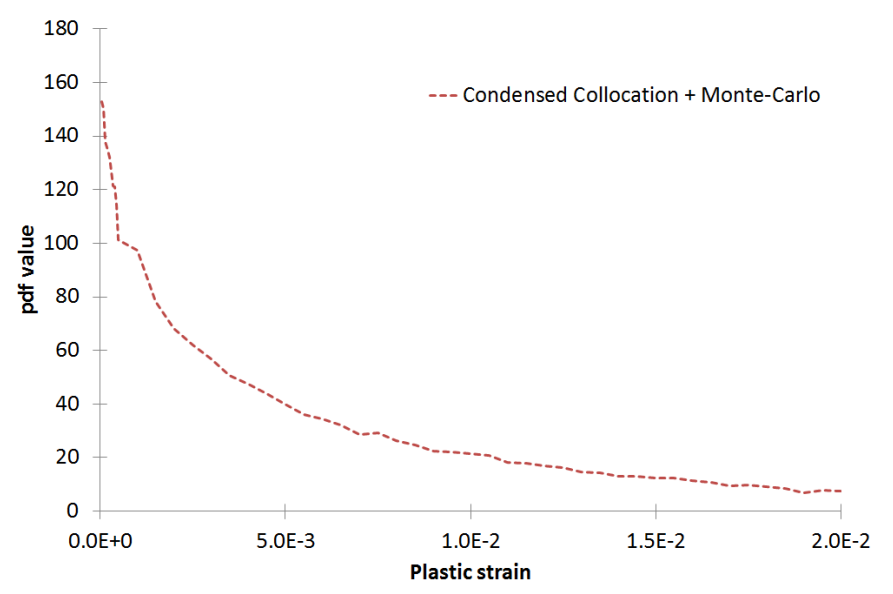

Figure 9: $\mathrm{PDF}$ of the plastic strain $\varepsilon_{p}$ on the 6 rvs variant of the steel bar.

\section{Application: Plain concrete beam under 4-point bending}

\subsection{Problem description}

This test case aims at validating the applicability of the proposed method on a typical structural nonlinear problem with uncertainties. The structure is a concrete beam undergoing four-point bending load. The objective is to build a metamodel of the mechanical behaviour and to extract a PDF for the maximum value of damage $D$ at the end of the loading.

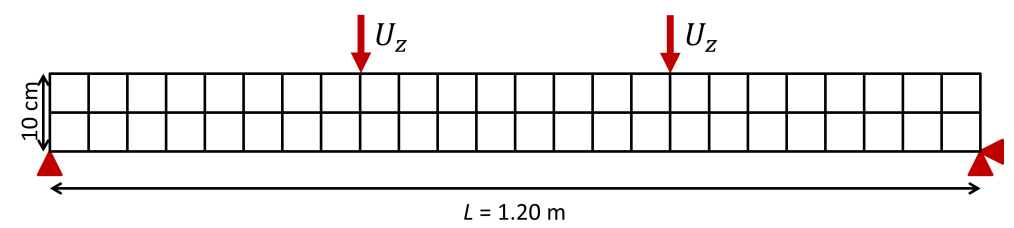

Figure 10: Scheme of the concrete beam with boundary conditions

The beam is modeled in two dimensions under the plane stress hypothesis. It is worth noting a 2D model is here only used for simplicity. The presented method can also be applied with 3D models. Fig. 10 presents the principle of the beam under loading. The beam is meshed uniformly with square bilinear elements (QUA4) of size $5 \times 5 \mathrm{~mm}$. Small elastic areas are used to avoid damage in the support points and loading 
areas. The loading is a vertical imposed displacement, up to $3 \times 10^{-4} \mathrm{~m}$, applied on two points on top of the beam, both located at $40 \mathrm{~cm}(L / 3)$ from the ends. This loading creates an homogeneous bending moment in the central third of the beam. The concrete is modeled using an isotropic damage model [26]. In this model, the damage evolution law writes :

$$
D(\kappa)=1-\frac{\kappa_{0}}{\kappa}\left(1-\alpha+\alpha \exp \left[-\beta\left(\kappa-\kappa_{0}\right)\right]\right)
$$

where $\kappa$ is the historical equivalent strain, $\kappa_{0}$ the damage threshold, $\alpha$ and $\beta$ parameters of the model, whose values are given in Table 4 .

Table 4: Material parameters for the concrete mechanical model

\begin{tabular}{|c|c|c|c|c|c|c|c|}
\hline \multicolumn{2}{|c|}{ Young's modulus* $\dagger$} & \multirow{2}{*}{ Poisson coeff. } & \multicolumn{2}{|c|}{ Damage threshold* } & $\alpha$ & $\beta$ & $\begin{array}{c}\text { compressive / tensile } \\
\text { strength ratio }\end{array}$ \\
\cline { 4 - 7 } Mean & Std. dev. & & Mean & Std. dev. & & & $k$ \\
\hline$E_{\mu}^{\prime}$ & $E_{\sigma}^{\prime}$ & $\nu$ & $\kappa_{\mu}$ & $\kappa_{\sigma}$ & $\alpha$ & $\beta$ & 11 \\
\hline $30 \mathrm{GPa}$ & $3 \mathrm{GPa}$ & 0.2 & $1 \times 10^{-4}$ & $5 \times 10^{-6}$ & 0.96 & 9000 & $k$ \\
\hline
\end{tabular}

To avoid pathological mesh dependency, and to ensure a better modeling of the damaged areas, the nonlocal stress-based method is applied [27] (nonlocal internal length $l_{C 0}=12 \mathrm{~mm}$ ). This allows the problem to be well-posed. Using this regularization method, the nonlocal equivalent strain $\tilde{\varepsilon}$ writes :

$$
\tilde{\varepsilon}(x)=\frac{\int_{\Omega} \bar{\varepsilon}(s) \phi(x-s) \mathrm{d} s}{\int_{\Omega} \phi(x-s) \mathrm{d} s}
$$

where $\bar{\varepsilon}$ is the equivalent strain, and $\phi$ is the stress-based weighting function, whose expression writes :

$$
\phi(x-s)=\exp \left(-\left(\frac{2\|x-s\|}{l_{C 0} \rho(x, \sigma(s))}\right)^{2}\right)
$$

where $l_{C 0}$ is the nonlocal internal length and $\rho$ a function of the stress state and geometry.

To model the heterogeneity of the mechanical properties in a material such as concrete, an heterogeneous field $C(x)$ is used for the Young's modulus. This parameter has been chosen as it creates an heterogeneity in both material strength and stiffness. All other mechanical parameters are considered homogeneous, the material spatial heterogeneity is represented by only one realization of an auto-correlated lognormal random field (of mean value $C_{\mu}=1$ and standard deviation $C_{\sigma}=0.067$ ), with an internal correlation length $l_{i}=12 \mathrm{~mm}$. It is to be noted that the nonlocal internal length $l_{C 0}$ and the internal correlation length $l_{i}$ of the random field are equal. Both those values are usually considered linked to the size of the aggregates, and have been taken equal.

Lognormal probability laws are often used in the field of mechanics to model uncertain material parameters, as they avoid creating negative (and nonphysical) values of material parameters. Therefore, lognormal 
distributions will be used to model the uncertain parameters. To model the uncertainty on the mechanical properties, a lognormal random variable $E^{\prime}$, with mean and standard deviation indicated in Table 4 weights the elastic modulus. The heterogeneous and random Young's modulus therefore writes :

$$
\forall x=\left(x_{1}, x_{2}\right) \in[0 ; H] \times[0 ; L], E\left(x, X_{1}\right)=E^{\prime}\left(X_{1}\right) \cdot C(x)
$$

Fig. 11 presents the random field $C(x)$ for the elastic modulus.

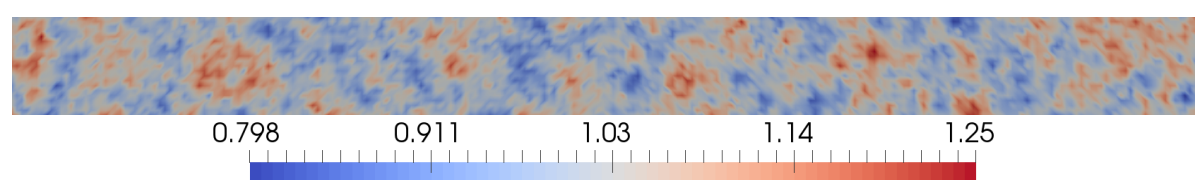

Figure 11: Field of (normalized) elastic modulus $C(x)$ in the concrete beam

A second r.v. is used to model the uncertainties on the mechanical properties: the damage threshold $\kappa_{0}$ used in the model, homogeneous in the beam, is a r.v. $\kappa_{0}\left(X_{2}\right)$. It also follows a lognormal law, with the parameters given in Table 4.

\subsection{Adaptive condensation and condensed collocation parameters}

On this test case, the adaptive condensation method [16] is applied on the simulations with 32 areas. Two random parameters are considered, namely the random elastic modulus $E^{\prime}\left(X_{1}\right)$ and the random damage threshold $\kappa_{0}\left(X_{2}\right)$. However, the damage threshold has no impact on the structure's elastic stiffness. Therefore, the metamodel for the condensed stiffness uses a polynomial decomposition of order $\left(n^{\prime}-1\right)=1$ (linear) on the single r.v. $X_{1}$ : condensed stiffnesses, and condensed area's displacements are interpolated from $n^{\prime}=2$ collocation points. Using the particular r.v.s of this problem, the approximation of the condensed stiffness matrices (Eq. (12)), condensed force vector (Eq. (13)), and condensed displacement fields in the condensed areas (Eq. (15)) can then be formulated as :

$$
\begin{aligned}
\hat{K}_{i}(X) & \simeq \sum_{p^{\prime}=1}^{n^{\prime}} \hat{K}_{i}^{p^{\prime}} \Phi_{p^{\prime}}^{E^{\prime}}\left(X_{1}\right) \\
\hat{F}_{i}^{\tau}(X) & \simeq \sum_{p^{\prime}=1}^{n^{\prime}} \hat{F}_{i}^{\tau, p^{\prime}}(X) \Phi_{p^{\prime}}^{E^{\prime}}\left(X_{1}\right) \\
u_{C i}^{\tau}(X) & \simeq \sum_{p^{\prime}=1}^{n^{\prime}} u_{C i}^{\tau, p^{\prime}}(X) \Phi_{p^{\prime}}^{E^{\prime}}\left(X_{1}\right)
\end{aligned}
$$

\subsection{Results}

On this application case, the results obtained by the standard stochastic collocation technique are compared to those of the condensed collocation method. 


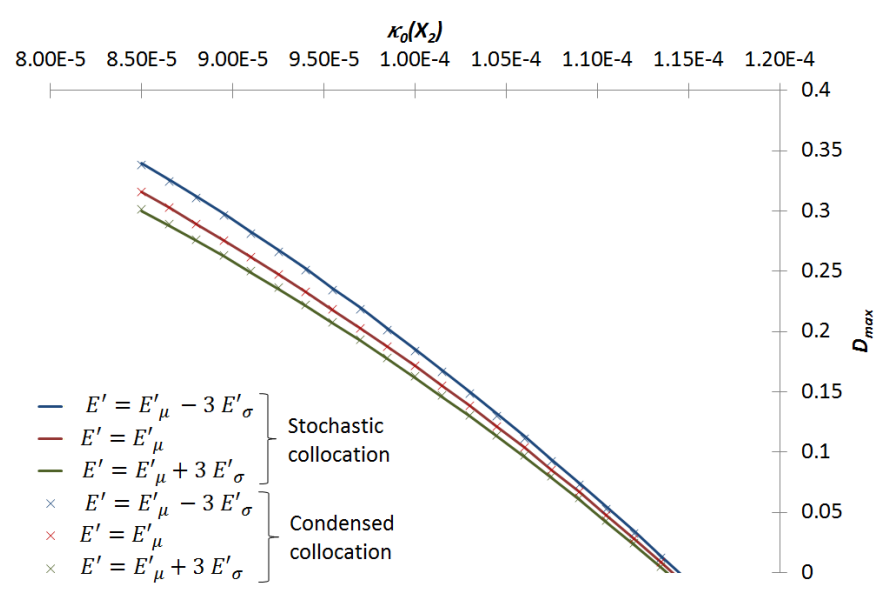

Figure 12: Metamodels of the maximal value $D_{\max }$ of the damage variable in the beam: Stochastic collocation vs. Condensed Collocation

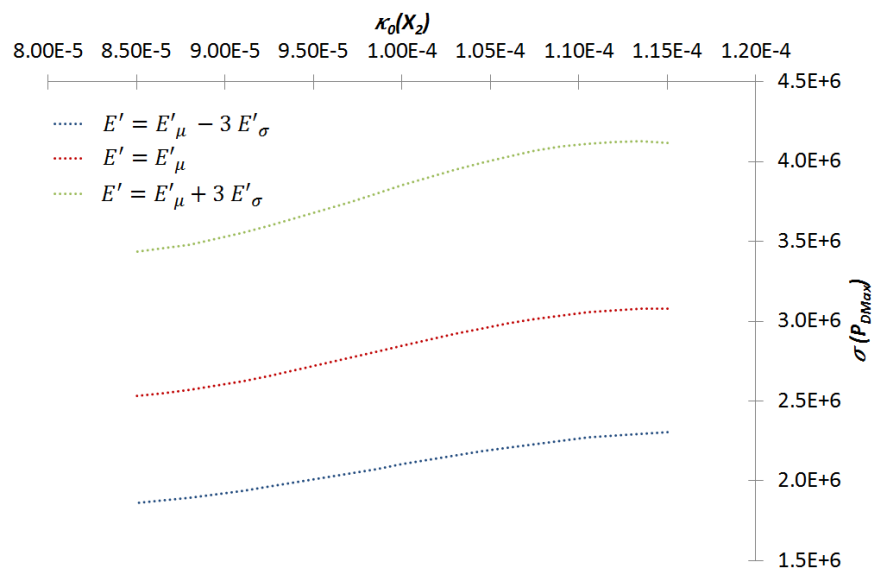

Figure 13: Metamodel of the equivalent stress $\sigma$ at the most damaged integration point: Condensed collocation.

Fig. 12 presents the metamodels generated through both techniques, to obtain an approximation of the maximal value $D_{\max }$ of damage in the beam at the end of the simulation. Both models use a 4 point collocation on the two r.v.s (and therefore, degree 3 polynomials on two variables). 16 condensed deterministic simulations are thus used to build this metamodel. The metamodels obtained from condensed collocation and standard collocation are in good agreement. On this application case (which includes 10,122 DOFs), using a higher-order metamodel with two r.v.s, the stochastic collocation method requires a computation time of $6,392 \mathrm{~s}$ to build the metamodel (on a standard dual-core workstation).

Fig. 14 presents the PDFs obtained with the metamodel from the condensed collocation method using both Monte-Carlo ( $10^{4}$ and $10^{5}$ realizations) and semi-analytical approaches. These PDFs are in good agreement. 


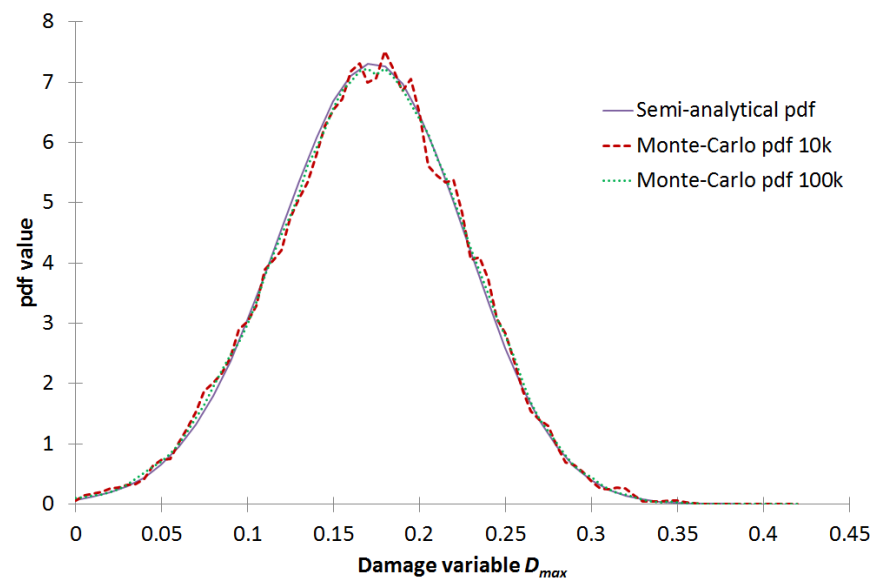

Figure 14: PDFs of the damage variable obtained with the condensed collocation method: Semi-analytical vs. Monte-Carlo $\left(10^{4}\right.$ and $10^{5}$ realizations)

Building the metamodel with the condensed collocation technique only requires $2,808 \mathrm{~s}$ of computation time on the same machine for equivalent results (Fig. 12-13), thus reducing the global computational load of the SFE calculation. As in the steel bar case, the reduction of computational load is related to the size of the problem, and especially of the non linear part of this problem. Using the semi-analytical formulation to compute the PDF of the quantity of interest provides a more accurate result (Fig. 14) and eliminates the computational cost associated to the MC method.

\section{Conclusions}

A condensed collocation approach has been presented for the SFE analysis of large-scale, locally nonlinear structures under uncertainties. It adopts the framework of stochastic collocation to build a polynomial expansion of the quantities of interest in a structural mechanical problem, and provides statistical results on these quantities, such as fragility curves, statistical moments or PDFs. In some cases, a semi-analytical technique allows to compute the PDFs of quantities of interest, more accurately and without the heavy data processing of MC approaches.

The proposed approach consists in a two-level coupling between SFEM and an adaptive condensation technique: it reduces both the cost of each deterministic simulation required in the SFE approach, and the cost of system reduction in the condensation technique. Each deterministic simulation necessary for SFEM is reduced using an adaptive, two-level condensation technique based on Guyan reduction, suited for nonlinear problems. The quantities required for this adaptive condensation method are approximated using a second level of polynomial expansion of smaller dimension on selected random parameters.

This method is applied on the numerical model of a steel tension rod with a localized defect, using a nonlinear mechanical model with one random parameter. On this test case, it reproduces the results of a 
state-of-the-art stochastic finite element method, in accordance with the analytical study, for a fraction of the computational cost. It also allows to perform a semi-analytical probabilistic study to obtain accurate statistical quantities such as the PDF of the plastic strain. When the number of random parameters increases, the second polynomial expansion allows to reduce largely the numerical cost of the condensation pre-processing. The method is also applied on a 4-point bending beam application case, where it builds a metamodel depending on two random variables. The metamodel is equivalent to the one obtained with stochastic collocation, for once again a fraction of the computational load.

Ongoing research will focus on several axes of development. In particular, an implementation using the same framework suited for nonlinear problems, but with a Galerkin-type SFE approach, similar to the work described in [14] would be most interesting. In fact, as the proposed implementation uses stochastic collocation, it is mostly usable for problems with large number $d$ of DOFs in the mechanical system and small number of r.v.s $n$. To build a $p$-order metamodel on such a system, the single stochastic collocation would require (in the linear case) $(p+1)^{n}$ solutions of linear systems of size $d$, for a total complexity in $\mathcal{O}\left((p+1)^{n} d^{2}\right)$. For the same order of approximation, a Galerkin-type polynomial chaos approach would require a single solution of a system of size $d(n+p) ! /(n ! p !)$, with a total complexity in $\mathcal{O}\left(((n+p) ! /(n ! p !))^{2} d^{2}\right)$. Therefore, a Galerkin-type SFEM using the same "condensed SFEs" approach would be faster on structural problems with large number of random variables, but would also require more memory. Also, the principle of the proposed method could be extended to dynamical problems, in particular with the use of condensed mass matrices in addition to stiffnesses.

\section{Acknowledgements}

The authors gratefully acknowledge the funding support from French National Research Agency through "MACENA" project (ANR-11-RSNR-012).

\section{References}

[1] A. Kolmogoroff, Grundbegriffe der Wahrscheinlichkeitsrechnung, Springer Berlin Heidelberg, Berlin, Heidelberg, 1933. URL http://link.springer.com/10.1007/978-3-642-49888-6

[2] N. Metropolis, S. Ulam, The Monte Carlo Method, Journal of the American Statistical Association 44 (247) (1949) 335341. doi:10.1080/01621459.1949.10483310.

URL http://amstat.tandfonline.com/doi/abs/10.1080/01621459.1949.10483310

[3] P. Spanos, R. Ghanem, Stochastic Finite Element Expansion for Random Media, Journal of Engineering Mechanics 115 (5) (1989) 1035-1053. doi:10.1061/(ASCE)0733-9399(1989)115:5(1035). URL http://dx.doi.org/10.1061/(ASCE) 0733-9399(1989)115:5(1035)

[4] H. G. Matthies, C. E. Brenner, C. G. Bucher, C. Guedes Soares, Uncertainties in probabilistic numerical analysis of structures and solids-Stochastic finite elements, Structural Safety 19 (3) (1997) 283-336. doi:10.1016/S0167-4730(97)000131. URL http://www.sciencedirect.com/science/article/pii/S0167473097000131 
[5] D. Xiu, Fast numerical methods for stochastic computations: a review, Communications in computational physics 5 (2-4) (2009) 242-272.

[6] G. Stefanou, The stochastic finite element method: Past, present and future, Computer Methods in Applied Mechanics and Engineering 198 (9-12) (2009) 1031-1051. doi:10.1016/j.cma.2008.11.007. URL http://www.sciencedirect.com/science/article/pii/S0045782508004118

[7] E. Patelli, H. M. Panayirci, M. Broggi, B. Goller, P. Beaurepaire, H. J. Pradlwarter, G. I. Schueller, General purpose software for efficient uncertainty management of large finite element models, Finite Elements in Analysis and Design 51 (2012) 31-48. doi:10.1016/j.finel.2011.11.003.

URL http://www.sciencedirect.com/science/article/pii/S0168874X11002174

[8] J. D. Arregui-Mena, L. Margetts, P. M. Mummery, Practical Application of the Stochastic Finite Element Method, Archives of Computational Methods in Engineering 23 (1) (2014) 171-190. doi:10.1007/s11831-014-9139-3. URL http://link.springer.com/article/10.1007/s11831-014-9139-3

[9] I. Babuska, R. Tempone, G. Zouraris, Galerkin Finite Element Approximations of Stochastic Elliptic Partial Differential Equations, SIAM Journal on Numerical Analysis 42 (2) (2004) 800-825. doi:10.1137/S0036142902418680. URL http://epubs.siam.org/doi/abs/10.1137/S0036142902418680

[10] J. Back, F. Nobile, L. Tamellini, R. Tempone, Stochastic Spectral Galerkin and Collocation Methods for PDEs with Random Coefficients: A Numerical Comparison, in: J. S. Hesthaven, E. M. Ronquist (Eds.), Spectral and High Order Methods for Partial Differential Equations, no. 76 in Lecture Notes in Computational Science and Engineering, Springer Berlin Heidelberg, 2011, pp. 43-62.

[11] G. Stefanou, M. Georgioudakis, M. Papadrakakis, Sequentially Linear Analysis of Structures with Stochastic Material Properties, in: Multiscale Modeling and Uncertainty Quantification of Materials and Structures, Springer International Publishing, 2014, pp. 19-32.

[12] H. Panayirci, G. Schueller, On the Capabilities of the Polynomial Chaos Expansion Method within SFE Analysis-An Overview, Archives of Computational Methods in Engineering 18 (1) (2011) 43-55. doi:10.1007/s11831-011-9058-5. URL http://dx.doi.org/10.1007/s11831-011-9058-5

[13] M. Guedri, N. Bouhaddi, R. Majed, Reduction of the stochastic finite element models using a robust dynamic condensation method, Journal of Sound and Vibration 297 (1-2) (2006) 123-145. doi:10.1016/j.jsv.2006.03.046.

URL http://www.sciencedirect.com/science/article/pii/S0022460X0600280X

[14] H. Panayirci, H. Pradlwarter, G. Schueller, Efficient stochastic structural analysis using Guyan reduction, Advances in Engineering Software 42 (4) (2011) 187-196. doi:10.1016/j.advengsoft.2011.02.004.

URL http://www.sciencedirect.com/science/article/pii/S0965997811000196

[15] R. J. Guyan, Reduction of stiffness and mass matrices, AIAA Journal 3 (2) (1965) 380-380. doi:10.2514/3.2874. URL http://dx.doi.org/10.2514/3.2874

[16] A. Llau, L. Jason, F. Dufour, J. Baroth, Adaptive zooming method for the analysis of large structures with localized nonlinearities, Finite Elements in Analysis and Design 106 (2015) 73-84. doi:10.1016/j.finel.2015.07.011.

URL http://www.sciencedirect.com/science/article/pii/S0168874X15001146

[17] CEA, Description of the finite element code Cast3m (2015).

URL http://www-cast3m.cea.fr/

[18] M. Rosenblatt, Remarks on a Multivariate Transformation, The Annals of Mathematical Statistics 23 (3) (1952) 470-472. doi:10.1214/aoms/1177729394.

URL http://dx.doi.org/10.1214/aoms/1177729394

[19] D. H. Fremlin, Measure theory, Vol. 2, Torres Fremlin, 2010.

[20] W. H. Press, S. A. Teukolsky, W. T. Vetterling, B. P. Flannery, Numerical recipes in FORTRAN, Cambridge University 
Press, 1992.

[21] P. D. Crout, A short method for evaluating determinants and solving systems of linear equations with real or complex coefficients, Electrical Engineering 60 (12) (1941) 1235-1240.

[22] J. Pellet, Dualisation of the boundary conditions, in: Code_Aster Open Source - General FEA software, 2011.

[23] J. Baroth, P. Bressolette, C. Chauviere, M. Fogli, An efficient SFE method using Lagrange polynomials: Application to nonlinear mechanical problems with uncertain parameters, Computer Methods in Applied Mechanics and Engineering 196 (45-48) (2007) 4419-4429. doi:10.1016/j.cma.2007.04.017.

URL http://www.sciencedirect.com/science/article/pii/S0045782507002307

[24] J. Humbert, J. Baroth, L. Daudeville, Probabilistic analysis of a pull-out test, Materials and Structures 43 (3) (2009) 345-355. doi:10.1617/s11527-009-9493-z.

URL http://link.springer.com/article/10.1617/s11527-009-9493-z

[25] European Committee for Standardization, EN 1992-1-1 Eurocode 2: Design of concrete structures - Part 1-1: General rules and rules for buildings, CEN, Brussels, 2005.

[26] R. H. J. Peerlings, R. De Borst, W. A. M. Brekelmans, M. G. D. Geers, Gradient-enhanced damage modelling of concrete fracture, Mechanics of Cohesive-Frictional Materials 3 (1998) 323-342.

[27] C. Giry, F. Dufour, J. Mazars, Stress-based nonlocal damage model, International Journal of Solids and Structures 48 (25-26) (2011) 3431-3443. doi:10.1016/j.ijsolstr.2011.08.012.

URL http://www.sciencedirect.com/science/article/pii/S002076831100299X 